\title{
METALOPROTEINASAS DE LA MATRIZ EXTRACELULAR Y LIPOPRO- TEINAS DE ALTA DENSIDAD EN INDIVIDUOS JÓVENES
}

\section{EXTRACELLULAR MATRIX METALLOPROTEINASES AND LIPOPROTEINS OF HIGH DEN- SITY IN YOUNG INDIVIDUALS}

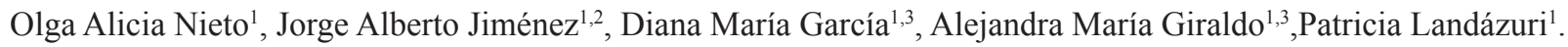

${ }^{1 .}$ Grupo de Investigación en Enfermedades Cardiovasculares y Metabólicas. Doctorado en Ciencias Biomédicas. Facultad de Ciencias de la Salud. Universidad del Quindío.

2. Programa de Química. Facultad de Ciencias Básicas y Tecnologías. Universidad del Quindío.

3. Programa de Educación Física. Facultad de Ciencias Educación. Universidad del Quindío.

4. Programa de Licenciatura en Biología y Educación ambiental. Facultad de Educación. Universidad del Quindío. Armenia-Quindío, Colombia.

Recibido: POR CONFIRMAR

Aceptado: POR CONFIRMAR

*Correspondencia del autor. Universidad del Quindío, Av. Bolivar calle 12 norte Armenia Quindío Colombia. e-mail: olgalicianieto@gmail.com

\section{RESUMEN}

Estudios realizados en el Quindío y Colombia describen que las HDL se encuentran por debajo de los niveles reconocidos como normales, sin embargo, se desconoce la situación respecto a apoA-I, MMP2 y MMP9; variables importantes en el reconocimiento del riesgo cardiovascular (RCV), sobre todo en las personas jóvenes.

Objetivo: determinar el perfil lipídico, las subfracciones de las HDL2 y HDL3, apoA-I, MMP2 y MMP9en suero y establecer una relación entre estas variables.

Métodos: el trabajo se realizó en estudiantes entre 18 y 29 años. Las subfracciones de HDL2 y HDL3 se midieron por el método de precipitación iónica, la apoA-I y las MMP2 y MMP9 se midieron por la técnica de Elisa.

Resultados: se encontró un perfil lipídico normal excepto por las HDL que fueron bajas con apoA-I normales. Los niveles de HDL2 y HDL3 fueron similares $(17,31 \pm 5,90$ y 17,99 $\pm 6,70 \mathrm{md} / \mathrm{dl})$ respectivamente, sin diferencias significativas entre ellos. Respecto a las MMP2 y MMP9, se encontraron niveles de 51,05 $\pm 5,69 \mathrm{ng} / \mathrm{ml}$ y 5,29 $\pm 3,03$ $\mathrm{ng} / \mathrm{ml}$, respectivamente. El análisis estructural evidenció una compleja interacción entre variables antropométricas, fisiológicas y bioquímicas: como la apoA-I y HDL2 y HDL3; entre TAS y TAD; entre MMP2 y MMP9. Entre IMC y apoA-I; el peso con HDL2, HDL3, TAS, TAD y MMP2. Y entre perímetro abdominal apoA-I, TAS, TAD, MMP2 y MMP9.

Conclusiones: este estudio revela un estado fisiológico y bioquímico normal, con interacciones entre variables que muestran una homeóstasis compatible con organismos jóvenes y saludables excepto por las HDL totales que son bajas.

Palabras Clave: jóvenes, HDL2, HDL3, MMP2, MMP9. 


\begin{abstract}
Studies in the Quindío and Colombia have reported that HDL is below that levels recognized as normal, however, the situation is unknown about the apoA-I, MMP2 and MMP9; important variables in the cardiovascular risk (CVR) recognition, especially in young people.

Objective: to determine the lipid profile, HDL2 and HDL3 subpopulations, apoA-I, MMP2 and MMP9 serum levels, and establish a relationship between these variables.

Methods: The study was conducted at students aged 18 to 29 years. The HDL2 and HDL3 subpopulations were measured by ion precipitation method, the ApoA-I, MMP2 and MMP9 were measured by ELISA.

Results: Lipid profile was normal, except for low HDL, despite having normal apoA-I. HDL2 and HDL3 subpopulations were found in similar levels $(17.31 \pm 5.90$ and $17.99 \pm 6.70 \mathrm{md} / \mathrm{dl})$, respectively, without significant differences between them. MMP2 and MMP9 were $51.05 \pm 5.69 \mathrm{ng} / \mathrm{ml}$ and $5.29 \pm 3.03 \mathrm{ng} / \mathrm{ml}$ respectively.

Structural analysis found a complex interaction between anthropometric, physiological and biochemical variables as: apoA-I, HDL2 and HDL3 subpopulations; between SBP and DBP; between MMP2 and MMP9. Between BMI and apoA-I; corporal weight with HDL2, HDL3, SBP, DBP and MMP2. And between waist circumference with apoA-I, SBP, DBP, MMP2 and MMP9.

Conclusions: This study reveals a normal biochemical and physiological state, with interactions between variables, which is compatible with healthy young organisms homeostasis, except fo rthe low total HDL.
\end{abstract}

Key words: young, HDL2, HDL3, MMP2, MMP9.

\section{INTRODUCCIÓN}

Cada vez hay mayor evidencia científica que los factores de riesgo desencadenantes de enfermedad cardiovascular se van acumulando desde la juventud, cuando las personas se sienten sanas(1), sin embargo, a pesar que la Organización Mundial de la Salud (OMS) ha hecho un llamado a los países en desarrollo para que pongan en práctica sistemas de monitoreo y prevención de las enfermedades cardiovasculares y sus causas subyacentes, el énfasis de los factores de riesgo para este grupo de población pone de relevancia en las políticas públicas el embarazo en adolecentes, el VIH, la violencia y la salud mental (1).

Las enfermedades cardiovasculares y entre ellas la hipertensión arterial, constituyen un problema de salud pública mundial y un importante factor de riesgo de morbilidad y mortalidad cardiovascular $(2,3)$; en el municipio de Armenia - Quindío y en Colombia, se identifica como la primera causa de muerte por enfermedades crónicas (4-5). En el municipio de Armenia, Quindío, la enfermedad hipertensiva, ocupó el primer lugar por mortalidad específica por enfermedades crónicas con una tasa de 13,02 en durante el año 2007 y subió en el año 2008 a 19,6 por 100.000 habitantes (4-6).

Una de las causas de hipertensión arterial (HTA) es el aumento de la resistencia vascular sistémica (RVS), la cual es un indicador del aumento del depósito de co- lágeno, fibrina y otras proteínas fibrilares en la matriz extracelular vascular (7). La matriz extracelular (MEC) en los seres humanos representa una red tridimensional que engloba todos los órganos, tejidos y células del organismo. Constituye un filtro biofísico de protección, nutrición e inervación celular y el terreno para la respuesta inmune, angiogénesis, fibrosis y regeneración tisular (8). La MEC está siendo remodelada constantemente por las metaloproteinasas de la MEC (MMP), una familia de endopeptidasas que hidrolizan sus constituyentes (9). Las MMP2 y MMP9 son enzimas de remodelación de la MEC, que se encuentran bajas en la HTA y aumentan hasta niveles similares al normal en respuesta al tratamiento antihipertensivo. Sin embargo, en pacientes con aumento de la RVS, las MMP no aumentan de la misma manera (10).

Otras causa del riesgo cardiovascular (RCV) es la dislipidemia, caracterizada por altos niveles de colesterol total (CT) y lipoproteinas de baja densidad (LDL) y bajos niveles de lipoproteinas de alta densidad (HDL), factores importantes en la génesis de la arterioesclerosis, que a su vez colabora con el aumento de la RVS y la hipertensión arterial. El aumento de las HDL se ha considerado como un factor protector en la HTA y en la enfermedad cardiovascular (ECV)(11). Asi mismo, las subfracciones de las HDL tiene un poder predictivo mayor que la cantidad presente en plasma, porque expresan cambios en la capacidad de transporte reverso de colesterol (un mecanismo a través del cual el exceso 
de colesterol regresa al hígado para ser eliminado), por lo cual las subfracciones de HDL se constituyen en un indicador de riesgo cardiovascular que se modifica en relación con otros factores asociados al estilo de vida (12).

Algunos miembros de las familias de las MMP (MMP3, MMP7 y MMP12) pueden remodelar las subpoblaciones de las HDL, específicamente HDL3, a través de la modificación proteolítica de la ApoA-I, la principal apoproteína de esta subpoblación, impidiéndole cumplir con su función de receptora de colesterol proveniente de las células (13).

Las HDL han demostrado ejercer un efecto protector contra las enfermedades cardiovasculares (ECV)(14). Sin embargo, estudios en población japonesa muestran un elevado porcentaje de ECV a pesar de altos niveles de HDL (15), esto sugiere que no sólo es importante un aumento en la concentración de las HDL sino en el tipo de las subpoblaciones o subfracciones que componen las HDL (16).

Sin embargo, no se conoce cuál es la situación de estas variables en personas jóvenes, sin enfermedades identificadas, que deberían ser el punto de partida para los programas de prevención del RCV y promoción de la salud, razón por la cual se llevó a cabo un estudio descriptivo para identificar las subfracciones de las HDL2 y HDL3, la apoA-I, las MMP2 y MMP y, establecer una relación entre estas variables en esta población.

\section{MATERIALES Y METODOS}

\section{Población y sujetos de estudio}

Se incluyeron en el estudio jóvenes (entre 19 y 29 años) estudiantes de medicina de ambos sexos, de la Universidad del Quindío, durante el segundo semestre de 2011, no hipertensos, no fumadores y sin otras enfermedades identificadas. Los estudiantes se citaron para informarles del estudio, posterior a la firma del consentimiento informado, se hizo una valoración inicial y registro de los datos y se tomó una muestra de sangre por punción venosa. Una vez se obtuvieron los resultados se registraron, procesaron y analizaron, se entregaron los resultados a los participantes; en todos los encuentros se hizo educación sobre los factores de riesgo cardiovascular. El estudio fue aprobado por el comité de bioética de la Universidad del Quindío.

\section{Definición de variables}

Se definió hipertensión como presión sistólica $>140$ y /o presión diastólica $>90 \mathrm{mmHg}$, tomada con el individuo sentado $(3,17)$. Con el peso y la talla se calculó el IMC en $\mathrm{Kg} / \mathrm{M}^{2}$, como sobrepeso se define un IMC entre 25,0 y $29,9 \mathrm{Kg} / \mathrm{M}^{2}$, y como obesidad un $\mathrm{IMC} \geq 30 \mathrm{Kg} / \mathrm{M}^{2}$.

\section{Análisis bioquímicos:}

Muestra sanguínea. Se obtuvo, después de doce horas de ayuno por punción venosa en dos tubos secos, el suero se obtuvo por centrifugación a $1000 \mathrm{~g}$ por $15 \mathrm{minu}-$ tos, a $4^{\circ} \mathrm{C}$, separado en tubos eppendorf y almacenado a $-20^{\circ} \mathrm{C}$ hasta su uso.

Perfil lipídico. El colesterol total y los triglicéridos se midieron por métodos enzimáticos comerciales. Para quienes tuvieron los triglicéridos inferiores a $400 \mathrm{mg} /$ dl, el colesterol en las lipoproteínas de baja densidad (C-LDL) se calcularon con la ecuación de Friedewald (18) y las VLDL $=\mathrm{TG} / 5$.

Subpoblaciones de HDL: Se analizaron en plasma, usando el método de Gidez y colaboradores (19) modificado por Berglund et al,(20) brevemente: El colesterol en las HDL totales se cuantificó por método enzimático en una alícuota del sobrenadante resultante después de la precipitación del plasma con heparina y $\mathrm{MgCl}_{2}$. En el resto del sobrenadante se cuantificaron las HDL3 después de la precipitación de las HDL2 con dextran sulfato. Las HDL2 se calcularon por la diferencia entre HDL total-HDL3. Para los debidos controles siempre se usó un set de tres muestras de plasma (rangos bajo, alto y medio) en cada ensayo.

Apolipoproteina A-I: Los niveles séricos de ApoA-I, se midieron por la técnica Elisa con anticuerpo específico, según instrucciones del fabricante (BindingSite ${ }^{\circledR}$ ). Metaloproteinasas: Los niveles séricos de MMP2 y MMP9, se midieron por la técnica de ELISA (R\&D systems $\left.{ }^{\circledR}\right)$, con anticuerpo específico siguiendo las indicaciones del fabricante.

\section{Análisis estadístico}

Se realizó un análisis descriptivo con los datos obtenidos y se calcularon las medias y la desviación estándar de las variables.

Para probar afirmaciones sobre el comportamiento de la población, que se evidencian a través de la muestra, frente a valores de referencia de los parámetros, se hicieron pruebas de hipótesis unilaterales verificando los supuestos de normalidad con la prueba de Kolmogorov Smirnov.

Se ajustaron modelos de regresión múltiple, con selección de variables, para analizar la interdependencia entre las variables cuantitativas.

Con los resultados de los modelos de regresión múltiple se construyeron diagramas de Motricidad-Dependencia, entre las variables cuantitativas utilizando técnicas 
Tabla 1. Variables epidemiológicas y bioquímicas de jóvenes no hipertensos

\begin{tabular}{|c|c|c|}
\hline VARIABLE & $\begin{array}{l}\text { PRIMERA MUESTRA } \\
n=19 \text { Estudiantes }\end{array}$ & VALORES DE REFERENCIA \\
\hline EDAD (años) & $21,42 \pm 3,5 \quad(19-29)$ & Jóvenes > 18 años \\
\hline TALLA (cms) & $1,66 \pm 0,11 \quad(1,52-1,90)$ & No aplica \\
\hline PESO (Kg) & $64,84 \pm 11,78$ (49-90) & No aplica \\
\hline IMC & $23,27 \pm 2,44(18,32-27,88)$ & 20 a 25 \\
\hline $\mathrm{PA}(\mathrm{cms})$ & $79,42 \pm 7,73 \quad(69-98)$ & $M<90-F<80$ \\
\hline $\mathrm{TA}(\mathrm{mm} / \mathrm{Hg})$ & $105 / 69$ & $120 / 80 \mathrm{~mm} \mathrm{Hg}$ \\
\hline TAS & $105 \pm 13,64(90-130)$ & \\
\hline TAD & $69,47 \pm 9,11 \quad(50-90)$ & \\
\hline CT (mg/dl) & $159,45 \pm 34,07(106,6-230,6)$ & $60-200 \mathrm{mg} / \mathrm{dl}$ \\
\hline TG $(\mathrm{mg} / \mathrm{dl})$ & $108,49 \pm 42,40(36,6-182,9)$ & Hasta $150 \mathrm{mg} / \mathrm{dl}$ \\
\hline $\mathrm{HDL}(\mathrm{mg} / \mathrm{dl})$ & $35,31 \pm 6,41 \quad(26,7-46,5)$ & $45-65 \mathrm{mg} / \mathrm{dl}$ \\
\hline HDL2 (mg/dl) & $17,31 \pm 5,90 \quad(8,29,64)$ & No aplica \\
\hline HDL 3 (mg/dl) & $17,99 \pm 6,70 \quad(0-29,72)$ & No aplica \\
\hline APOA-I (g/l) & $1,25 \pm 0,21(0,891-1,779)$ & $1,24-2,02$ \\
\hline MMP2 ng/ml) & $51,05 \pm 5,69 \quad(41,65-61,56)$ & No aplica \\
\hline MMP9 (ng/ml) & $5,29 \pm 3,03 \quad(1,38-13,01)$ & No aplica \\
\hline
\end{tabular}

IMC: Índice de masa corporal. PA: Perímetro abdominal. TA: Tensión arterial. TAS: Tensión arterial sistólica. TAD: Tensión arterial diastólica. CT: Colesterol total. TG: Triglicéridos. HDL: Lipoproteína: de alta densidad. HDL2: HDL subfracción2. HDL3: HDL subfracción3. apoA-I: Apolipoproteína A1 LDL: Lipoproteínas de baja densidad. VLDL: IA: Índice arterial. MMP2: Metaloproteinasa 2 de la matriz. MMP9: Metaloproteinasa 9 de la matriz.

de Análisis Estructural.

Para el procesamiento estadístico de los datos se utilizó el programa Statgraphics Centurion 15.

Para analizar la estructura de dependencia entre las variables se utilizó la técnica del análisis estructural (21). Para el Análisis Estructural se utilizó el software Mic Mac.

\section{RESULTADOS}

El estudio incluyó 19 personas jóvenes, 11 mujeres y 8 hombres, no fumadores y sin enfermedades identificadas. La tabla 1 muestra las variables epidemiológicas y bioquímicas de la población.

Los resultados muestran que la población a estudio tenía el IMC, PA, CT y TG normales. Con relación a las HDL los datos indican que estas lipoproteínas se en- 
cuentran por debajo de las cifras establecidas como normales, lo cual se confirmó con una prueba de hipótesis. No hubo diferencia significativa con respecto a los niveles de las subfracciones de HDL en esta población, así la concentración de las HDL3 fue de $17,99 \pm 6,70 \mathrm{mg} / \mathrm{dl}$ y las HDL2 de 17,31 $\pm 5,90 \mathrm{mg} / \mathrm{dl}$. La apoA-I se encontró en niveles establecidos como normales, $1,25 \pm 0,21$ $\mathrm{g} / \mathrm{l}$, es decir, los jóvenes universitarios de este estudio presentan apoA-I normales y HDL bajas.

En este trabajo también se describen los niveles de las MMP, conocidas como gelatinasas: la MMP2 se encon-

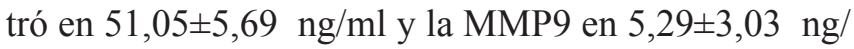
$\mathrm{ml}$.

Relación entre los niveles séricos de las MMP2, MMP9, las subfracciones de HDL y ApoA-I.

Como puede observarse en la tabla 2, A través de un modelo de regresión múltiple, se analizó la interdependencia entre variables cuantitativas. Los resultados muestran que en los jóvenes no hipertensos, no fumadores, sin enfermedades identificadas, se presenta una gran interacción entre las variables, tanto fisiológicas (TAS, TAD, edad) como bioquímicas; donde cada una de ella interactúa en una relación de dependencia con respecto a las otras variables; así por ejemplo, la MMP9 depende en un $34 \%$, de la TAS, el peso y el perímetro abdominal, las tres con un valor de P altamente significativo. La dependencia más alta se encontró en la TAS la cual depende significativamente en un 92,69\% de la MMP9, las TAD y la HDL2 principalmente. Las otras dependencias entre variables pueden visualizarse en la tabla 2 y en la figura 1 .

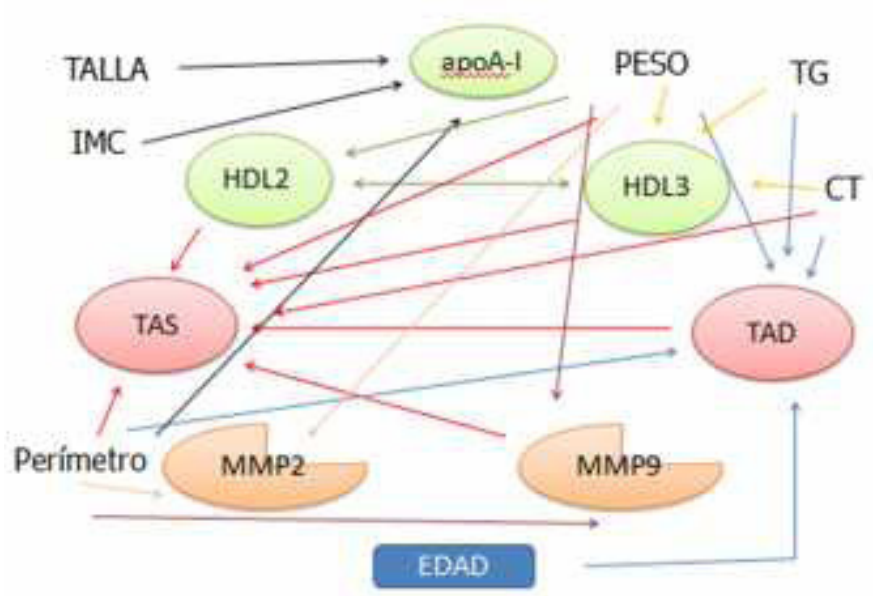

Figura 1. Relaciones fisiológicas y bioquímicas en jóvenes Nieto OA. 2012.
Como puede observarse en la figura 1, la regresión múltiple evidencia que existe una compleja interacción entre las variables antropométricas, fisiológicas y bioquímicas, tales como: entre la apoA-I y las subfracciones de HDL2 y HDL3; entre las TAS y la TAD; entre las MMP2 y MMP9. Entre IMC con la apoA-I; el peso con la HDL2, HDL3, la TAS y la TAD y, la MMP2. Y entre el perímetro abdominal con variables como la apoA-I, la TAS, la TAD, la MMP2 y MMP9.

Cuando se hizo el análisis de motricidad y dependencia entre variables se observó que, en los jóvenes de este estudio, las variables de mayor poder y motricidad son la TAS y la TAD, con una fuerte influencia entre ellas. La TAD a su vez mueve a la apoA-I, con una influencia relativamente fuerte, como puede verse en la figura 2 .

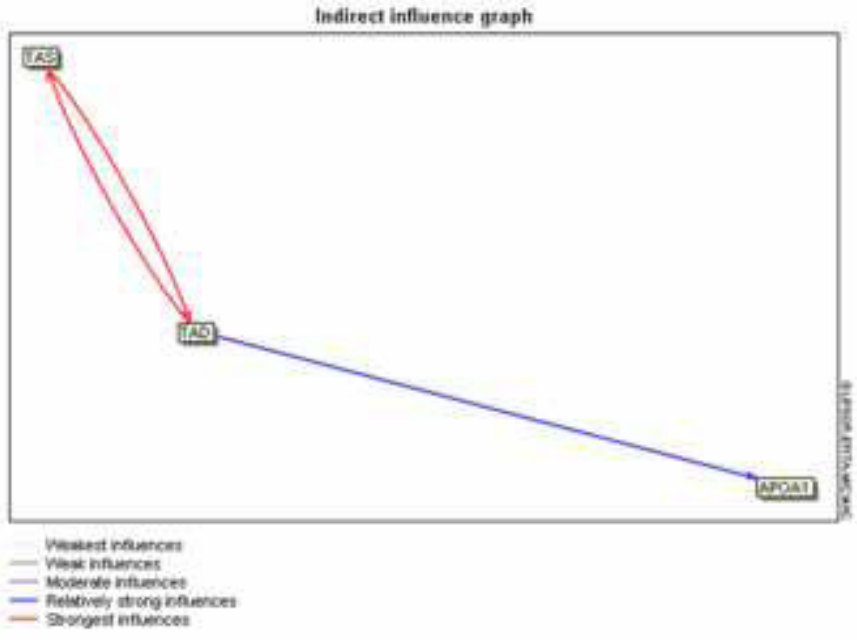

Figura 2. Motricidad y dependencia de variables bioquímicas y fisiológicas en jóvenes

\section{DISCUSION}

Un acúmulo importante de evidencia científica relaciona a las MMP con las enfermedades cardiovasculares $(22,23)$, así, se ha demostrado una asociación de la rigidez vascular y el desarrollo de arterosclerosis con los niveles séricos de la MMP9 (24). Por otro lado también hay evidencia que relaciona la estructura y función de las HDL con las metaloproteinasas, siendo estas enzimas que las que remodelan el tamaño y composición de apoA-I en las HDL (13). En el presente trabajo se estudiaron las MMP9, MMP2, ApoA-I y las subfracciones HDL para establecer sus niveles séricos y las interrelaciones que entre ellas ocurren en población joven sin enfermedad cardiovascular diagnosticada.

Los resultados muestran unos niveles de MMP9 y MMP2, mucho menores a los encontrados por Yasmin 
Tabla 2. Relación y significancia mediante un modelo de regresión múltiple

\begin{tabular}{|c|c|c|c|c|c|}
\hline \multicolumn{6}{|l|}{ VARIABLES } \\
\hline DEPENDIENTES & MMP & EDAD & T.A. & HDL & OTROS \\
\hline TAS & MMP9 & & TAD & HDL2 & $\mathrm{CT}-\mathrm{TG}$ \\
\hline \multirow[t]{3}{*}{$R^{2}=92,69 \%$} & $P=0,0050$ & & $P=0,171$ & $P=0,0043$ & PA - PESO \\
\hline & & & & HDL3 & \\
\hline & & & & $P=0,0054$ & \\
\hline TAD & & EDAD & & & $\mathrm{CT}-\mathrm{TG}$ \\
\hline \multirow[t]{2}{*}{$R^{2}=83,64 \%$} & & $P=0,0255$ & & & PA - PESO \\
\hline & & & & & TALLA \\
\hline HDL2 & & & & HDL3 & PESO \\
\hline$R^{2}=52,56 \%$ & & & & $P=0,0132$ & $P=0,0285$ \\
\hline HDL3 & & & & HDL2 & CT-TG \\
\hline $\mathrm{R}^{2}=74,53 \%$ & & & & $P=0,0003$ & PESO \\
\hline APOA-I & \multicolumn{5}{|c|}{ IMC: $P=0,01334-P A: P=0,0089-$ TALLA: $P=0,0231$} \\
\hline \multicolumn{6}{|l|}{$R^{2}=45,92 \%$} \\
\hline MMP2 & & & & & $P A=0,0069$ \\
\hline$R^{2}=45,43 \%$ & & & & & PESO $=0,0221$ \\
\hline MMP9 & & & TAS & & $P A=0,0208$ \\
\hline $\mathrm{R}^{2}=34,38 \%$ & & & $P=0,0253$ & & $\mathrm{PESO}=0,0390$ \\
\hline
\end{tabular}

IMC: Índice de masa corporal. PA: Perímetro abdominal. TA: Tensión arterial. TAS: Tensión arterial sistólica. TAD: Tensión arterial diastólica. CT: Colesterol total. TG: Triglicéridos. HDL: Lipoproteínas de alta densidad. HDL2: HDL subfracción2. HDL3: HDL subfracción3. apoA-l: Apolipoproteína A1. LDL: Lipoproteínas de baja densidad. VLDL: IA: Índice arterial. MMP2: Metaloproteinasa 2 de la matriz. MMP9: Metaloproteinasa 9 de la matriz.

y colaboradores (24), para pacientes con hipertensión aislada y su grupo control con un promedio de edad de ambos de 68 años. Esta diferencia puede obedecer a múltiples razones, entre ellas la diferencia de edad, la base genética de la población estudiada y la estandarización de los métodos de medida de las MMP. Para determinar si la concentración de las MMP halladas aquí son los niveles de nuestra población es necesario un estudio más amplio.

Las HDL comprenden un grupo heterogéneo de lipoproteínas que pueden clasificarse por tamaño según el método de separación (25); por precipitación iónica se obtienen dos subfracciones las HDL3 pequeñas y densas cuyo contenido es pobre en ésteres de colesterol y las HDL2 más grandes y menos densas, con alto contenido en esteres de colesterol y triglicéridos. Ambas subfracciones tienen a la ApoA-I como proteína prin- cipal (25). En la circulación estas subfracciones intercambian contenidos entre sí debido a las acciones de diversas enzimas y cofactores incluidas en el transporte reverso del colesterol (25).

Se ha demostrado que la remodelación de las subfracciones se ve interferida en diversas patologías y que el cambio en ellas por ejemplo es predictor de la progresión de la enfermedad coronaria (26), o de presencia y la severidad de la aterosclerosis (27).

Este resultado confirma estudios anteriores de población de riesgo cardiovascular en la región (28) en los cuales se demostraron HDL por debajo de las cifras normales. Pero sólo hasta ahora, el presente estudio muestra valores de concentración de las subpoblaciones de HDL2 y HDL3, las cuales para esta población de jóvenes fueron similares, sin embargo, en diversos estudios en individuos con enfermedad coronaria se encuentran diferen- 
cias significativas entre estas dos subpoblaciones (26, 27). Es posible que el desbalance en el equilibrio de estas subpoblaciones sea una de las múltiples causas de la EC como lo demuestran los estudios antes referidos. $\mathrm{Y}$ que en esta población joven y aparentemente sana no se muestre aún este desequilibrio sin embargo, por el tamaño de la población estudiada no es posible extraer esta conclusión.

Los otros parámetros del perfil lipídico, tales como el CT, TG, LDL, se encontraron en límites normales. Sin embargo, el índice arterial (IA), que expresa la relación $\mathrm{CT} / \mathrm{HDL}$, se encontró en 4,54 (niveles normales $<3,54)$. Es decir, a pesar de tener el CT, TG, LDL y apoA-I normales, la población de jóvenes universitarios de este estudio tiene ya aumento del riesgo cardiovascular, por su baja concentración en HDL.

Sobre las metaloproteinasas, la MMP2 ó gelatinasa A, corta una gran variedad de sustratos y su actividad biológica está asociada muchos procesos fisiopatológicos que incluyen la enfermedad cardiovascular, en la cual juega un rol de pivote entre la vasoconstricción y la vasodilatación (29). A la MMP9 o gelatinasa B, algunos estudios la muestran como indicador de RCV, al hacer disrupción de la placa ateroesclerótica (29). En este estudio se quería establecer una línea base de las MMP2 y MMP9, que hasta donde se conoce no habían sido medidas antes en esta población. La MMP2 se encontró en $51,05 \pm 5,69 \mathrm{ng} / \mathrm{ml}$ y la MMP9 en $5,29 \pm 3,03 \mathrm{ng} / \mathrm{ml}$. Valores bajos cuando se les compara con los hallados por Yasmin y colaboradores (24) en pacientes con hipertensión (151,7 y 119,5 ng/ml respectivamente), no fue posible la comparación de estos valores con otros en poblaciones jóvenes.

Sobre la relación entre las MMP y las HDL, hay referencias (13), que muestran como las MMP3,7,12 cortan la apoA-I y otros autores $(30,31)$, muestran como las HDL, previenen la oxidación de las LDL y aumentan la regulación de la MMP2 (32).

El análisis de regresión múltiple y el análisis estructural y de motricidad en los datos de estas variables para la población del presente estudio mostraron una gran interacción entre las variables que son señaladas en la tabla 2 y la figura 1.

Se evidencia a través del análisis estadístico que hay una compleja interacción entre las variables antropométricas, fisiológicas y bioquímicas, tales como: entre la apoA-I y las subfracciones de HDL2 y HDL3; entre las TAS y la TAD; entre las MMP2 y MMP9. Entre IMC con la apoA-I; el peso con la HDL2, HDL3, la TAS y la TAD y, la MMP2. Y entre el perímetro abdominal con variables como la apoA-I, la TAS, la TAD, la MMP2 y
MMP9.

Algunas de estas relaciones ya han sido reconocidas con anterioridad en otros grupos etáreos, en los cuales, cambios en variables como el peso influyen favorablemente en las subclases de lipoproteínas (33).

\section{CONCLUSIONES}

Se concluye que la población de jóvenes universitarios de este estudio, presenta cifras de Presión Arterial e IMC normales; así mismo son normales los niveles de CT y TG. Las HDL son bajas $(35,31 \mathrm{mg} / \mathrm{dl})$ a pesar de tener apoA-I normales $(1,25 \mathrm{~g} / \mathrm{L})$ y las subfracciones de HDL2 y HDL3 tenían niveles similares (17,31 mg/dl y $17,99 \mathrm{mg} / \mathrm{dl}$ respectivamente). Respecto a las MMP2 y MMP9, medidas por primera vez en esta población, se encontró la MMP2 en 51,05 $\pm 5,69 \mathrm{ng} / \mathrm{ml}$ y la MMP9 en $5,29 \pm 3,03 \mathrm{ng} / \mathrm{ml}$.

Se estableció un modelo de relación y dependencia entre las variables, que mostró una gran complejidad y dependencia entre ellas de un 34 a 92\%.

Se evidenció a través del análisis estadístico que hay una compleja interacción entre las variables antropométricas, fisiológicas y bioquímicas, tales como: entre la apoA-I y las subfracciones de HDL2 y HDL3; entre las TAS y la TAD; entre las MMP2 y MMP9.Entre IMC con la apoA-I; el peso con la HDL2, HDL3, la TAS y la TAD y, la MMP2. Y entre el perímetro abdominal con variables como la apoA-I, la TAS, la TAD, la MMP2 y MMP9. 


\section{AGRADECIMIENTOS}

A los estudiantes motivados por la investigación que participaron en este proyecto.

Al Grupo de apoyo en Investigación y asesoría en Estadística.

Financiación: Este proyecto se llevó a cabo con la financiación de la Vicerrectoría de Investigaciones y el Doctorado en Ciencias Biomédicas dela Universidad del Quindío.

\section{BIBLIOGRAFÍA}

1. $\quad$ OMS. 2011. Riesgos para la salud de los jóvenes. Nota descriptiva N³45 Agosto de 2011.

2. Kearney PM, Whelton M, Muntner P, Whelton PK, He J. Global burden of hypertension: Analysis of worldwide data. Lancet. 2005; 365:217-23.

3. LudovitPaulis and Thomas Unger. Novel therapeutic targets for hypertension. Nature Reviews/Cardiology. 2010; 7:431-41.

4. Ministerio de Salud. 2000. Guía de atención de la hipertensión Arterial. Resolución 412. Bogotá.

5. Alcaldía de Armenia. 2008. Situación de salud en Armenia. Indicadores básicos 2006-2007. Lupita, la que nos ayuda a ver. P10-15.

6. Alcaldía de Armenia. 2009. Boletín epidemiológico Armenia 2008. Lupita, la que nos ayuda a ver. P6-7.

7. Briones AM, Arribas SM and Salaices M. Role of extracelular matrix in vascular remodeling of hypertension. Curr Opin Nephrol Hypertens. 2010; 19:187-94.

8. Noguera R, Nieto OA, Tadeo I, Fariñas F and Álvaro T. Extracellular matrix, biotensegrity and tumor microenvironment. An update and overview. HistolHistopathol. 2012; 27: 693-705.

9. Rawlings ND, Barrett AJ, and Bateman A. MEROPS: the database of proteolytic enzymes their substrates and inhibitors. Nucleic Acids Res. 2012; 40:D343-D50.

10. Zervoudaki A, Economou E, Stefanadis C, Pitsavos C, Tsioufis K, Aggeli C, et al. Plasma levels of active extracelular matrix metalloproteinases 2 and 9 in patients with essential hypertension before and after antihypertensive treatment. Journal of Human Hypertension. 2003; 17:119-24.

11. Davidson WS, Gangani RA, Silva D, Chantepie S, Lagor WR, Chapman MJ, Kontush A. Proteomic Analysis of Defined HDL Subpopulations Reveals Particle-Specific Protein Clusters Relevance to Antioxidative Function. Arterioscler Thromb Vasc Biol. 2009; 29:870-76.

12. McPherson PAC, Young IS, McKibben B, McEneny J.High density lipoprotein subfractions: isolation, composition, and their duplicitous role in oxidation. J. Lipid Res. 2007; 48 86-95.

13. Lindstedt L, Saarinen J, Kalkkinen N, Welgus H, Kovanen PT.Matrix metalloproteinases-3,7, and 12, but Not 9, reduce High Density Lipoprotein-induced cholesterol efflux from Human macrophage foam cells by truncation of the carboxyl terminus of Apolipoprotein A-I. J Biol Chem. 1999;274:22627-34.

14. Gordon A. F. 2010. The complexity of HDL. Biochimica et Biophysica Acta 1801. 1286-93

15. Zhong S, Sharp DS, Grove JS, Bruce C, Yano K, Curb JD, Tall AR. Increased coronary heart disease in Japanese-American men with mutation in the cholesteryl ester transfer protein gene despite increased HDL levels. J. Clin. Invest.1996; 97:2917-23.

16. Chei CL, Yamagishi K, Kitamura A, Kiyama M, Imano H, Ohira T, et al. High-density Lipoprotein Subclasses and Risk of Stroke and its Subtypes in Japanese Population: The Circulatory Risk in Communities Study. Stroke. 2013; 44:327-33.

17. Chobaniam AV, Bakris GL, Black HR, Cushman WC, Green LA, Izzo JL et. al, and the National High Blood Pressure Education Program Coordinating Committee. The Seventh Report of the Joint National Committee on Prevention, Detection, Evaluation, and Treatment of High Blood Pressure. The JNC 7 Report. JAMA. 2003; 289:2560-72.

18. Friedewald WF, Levy RI, Fredrickson DS. Estimation of the concentration of low-density lipoprotein cholesterol in plasma without use of the preparative ultracentrifuge. Clin Chem. 1977; 18:499-502.

19. Gidez LI, Miller GJ, Burstein M, Slagle S, Eder HA. Separation and quantitation of human plasma high density lipoproteins by a simple precipitation procedure. J Lipid Res. 1982; 23:1206-23.

20. Berglund L, Oliver EH, Fontanez N, Holleran S, Matthews K, Roheim PS, et al. HDL-subpopulation patterns in response to reductions in dietary total and saturated fat intakes in healthy subjects. Am J ClinNutr. 1999; 70:992-1000. 
21. Mojica Sastoque, F. La prospectiva, técnicas para visualizar el futuro. 1991. Legis. Bogotá. 144 pag.

22. Newby AC. Dual role of matrix metalloproteinases (matrixins) in intimal thickening and atherosclerotic plaque rupture. Physiol Rev. 2005; 85:1-31.

23. Rodríguez, D, Morrison CJ, Overall, CM. Matrix metalloproteinases: What do they not do? New substrates and biological roles identified by murine models and proteomics. Biochimica et Biophysica Acta. 2010; 1803:39-54.

24. Yasmin, Wallace S, McEniery CM, Dakham Z, Pusalkar P, Maki- Petaja K, et al. Matrix metalloproteinase-9 (MMP-9), MMP-2, and serum elastase activity are associated with systolic hypertension and arterial stiffness. Arterioscler Thromb VascBiol. 2005; 25:372.

25. Singh IM, Shishehbor MH, Ansell BJ. High-Density Lipoprotein as a Therapeutic Target. JAMA. 2007; 298:786-98.

26. Asztalos BF, Batista M, Horvath KV, Cox CE, Dallal GE, Morse JS, Brown GB, Schaefer EJ. Change in $\alpha 1$ HDL Concentration Predicts Progression in Coronary Artery Stenosis. Arterioscler Thromb Vasc Biol. 2003; 23:847-52

27. Watanabe H, So“derlund S, Soro-Paavonen A, HiukkaA, LeinonenE,Alagona C, Salonen R, Tuomainen TP, EhnholmC, Jauhiainen M, Taskinen MR. Decreased High-Density Lipoprotein (HDL) Particle Size, Pre $\beta$, and Large HDL Subspecies Concentration in Finnish Low-HDL Families Relationship With Intima-Media Thickness. Arterioscler Thromb Vasc Biol. 2006; 26:897-902.

28. Landázuri P, Loango N, Gallego ML. Cardiovascular risk factors in first-degree relatives of patients with hypertension. Colombia Médica. 2011; 42:17-25.

29. Sbardella D, Frasciglione GF, Gioia M, CiaccioCh, Tundo GR, Marini S, Coletta M. Human matrizmetalloproteinases: An ubiquitarian class of enzymes involved in several pathological processes. Molecular Aspect of Medicine. 2012; 33:119-208.

30. Norata GD and Catapano AL. Molecular mechanisms responsible for the anti inflammatory and protective effect of HDL on the endothelium. Vascular Health and Risk Management. 2005; 1:119-29.

31. Xiao-Ping X, Meisel SR, Ong JM, Kaul S, Cercek B, Rajavashisth TB, et.al. Oxidized Low-Density Lipoprotein Regulates Matrix Metalloproteinase-9 and Its Tissue Inhibitor in Human Monocyte-Derived Macrophages. Circulation. 1999; 99:993-998.

32. Robbesyn F, Augé N, Cantero VC, Barbaras R, Negre-Salvayre A, and Salvayre R. High-Density Lipoproteins Prevent the Oxidized Low-Density Lipoprotein-Induced ndothelial Growth Factor Receptor Activation and Subsequent Matrix Metalloproteinase-2 Upregulation. Arterioscler Thromb Vasc Biol. 2005; 25:1206-12.

33. Mäntyselkä P, Kautiainen H, Saltevo J, Würtz P, Soininen P, Kangas AJ, et al. Weight change and lipoprotein particle concentration and particle size: a cohort study with 6.5-year follow-up. Atherosclerosis. 2012; 223:239-43. 